\title{
New, fully immersive virtual event
}

A new online virtual event is set to break the mould this autumn as Dentsply Sirona announces the launch of Let's Talk Live Decoding Digital Dentistry, which will take place on 8-9 October.

The event will be a fully immersive, two-day extravaganza, unlike any of its contemporaries, and will include the chance for one delegate to win a truly incredible prize - a Primescan intraoral scanner.

Throughout the two days, delegates will be treated to a total of 12 interactive sessions, featuring high-calibre national and international speakers who will present an inspiring range of dental topics.

'Interactive' is a key theme of Decoding Digital Dentistry and each session will be followed by a Round Table Live Q\&A, creating a forum discussion, during which delegates will have the opportunity to delve deeper into the subject and start a conversation with the speaker and other delegates.

There will also be the chance to chat with colleagues in online networking rooms, recreating opportunities that are normally found at a live conference. Related content will also be available online for a period after the event, available exclusively to those who register.

In addition to the presentations and live discussions, there will also be commercial sessions. These will be available on demand and can be supplemented with one-to-one consultations with Denstply Sirona experts, which can be booked in advance.

Dentsply Sirona's Primescan intraoral scanner is the linchpin of digital dentistry and for one lucky delegate it will become an important addition to their surgery - for free! Primescan features advanced technology that produces photorealistic, highly accurate data up to a depth of $20 \mathrm{~mm}$. Fast, hygienic and comfortable for patients, the winner will be the proud owner of one of the most advanced pieces of digital impression technology, making faster diagnosis and more accurate, predictable results a reality.

Tickets to Let's Talk Live Decoding Digital dentistry are $£ 80+$ VAT per delegate for the two days or a group ticket of up to five delegates is available for $£ 350+$ VAT.

Register now at https://cvent.

me/8wA1wR.

Save the date - 8/9 October 2021. Look out for more details, coming soon.

To find out more about Primescan, visit www.dentsplysirona.com/primescan.

\section{New platform for dental implant professionals}

PEERS is the Platform for Exchange of

Experience, Research and Science, which has been created to promote a productive and supportive partnership between Dentsply Sirona and dental implant professionals.

History has shown that development and the ability to innovate is most successful when we work together with professionals to share ideas and knowledge. PEERS is a platform fuelled by continual investment in research, learning and professional development to further these crucial ingredients for success.

The invitation-only PEERS group seeks to enable networking regionally, nationally and globally. It is a platform for meetings and events, product information, expertto-expert courses, speaker development, scientific research updates and much more.

Members of PEERS benefit from many exciting opportunities:

- To meet with national opinion leaders in small exclusive groups

- To access specially developed PEERS training and education programmes, events and seminars

- To participate in specialised working groups and various projects
- To be the first to receive news about the latest developments in implant dentistry

- To exchange ideas and opinions with like-minded professionals.

PEERS offers a platform for its members to share valuable knowledge and expertise with other members of the network. Dentsply Sirona takes care of all the organisation of events and meetings, allowing members to focus on the exclusive content, networking and furthering their own professional development.

Dentsply Sirona's $40+$ years of experience in all fields and technologies of implant dentistry and their dedication to longterm clinical evidence have resulted in unique industry insight and the ability to provide implant workflow solutions that offer simplified procedures and improved clinical efficacy.

PEERS is an opportunity to share this rich knowledge and experience more effectively with dental implant professionals and create a partnership that will lead to long-lasting and life-changing results for patients all over the world.

To learn more, download the online PEERS brochure from https://bit.ly/3sxAJQR.

\section{Zygomatic implant course}

The ZAGA Centre Manchester is delighted to be hosting the inaugural ZAGA Proficient Course in the UK, delivered in collaboration with Dr Carlos Aparicio - the creator of the ZAGA-Straumann Zygomatic system.

The intensive three-day programme teaches the evidence-based techniques, materials and protocols of the ZAGA method, which is designed to provide predictable long-term results with zygomatic implants. Delegates will explore the opportunities, benefits and limitations of the method, gaining hands-on experience with the Straumann ZAGA implants and learning related prosthetic rehabilitation and immediate loading of quad zygomatic implant treatment.

The course is presented by some of the most experienced clinicians in the field. In addition to Dr Carlos Aparicio, delegates will learn from Dr Rubén Davó - one of the most renowned zygomatic implant experts around the world - and Professor Cemal Ucer - Specialist Oral Surgeon and director of the Centre for Oral and Maxillofacial Dental Implant Reconstruction and ZAGA Centre Manchester.

Book your place today at https:// www.tickettailor.com/events/ zygomazagacenters/537408. 